\title{
Objective estimates of the probability of developing hypothyroidism following radioactive iodine treatment of thyrotoxicosis
}

\author{
Aftab M Ahmad ${ }^{1,3}$, Munir Ahmad ${ }^{2}$ and Eric T Young ${ }^{3}$ \\ ${ }^{1}$ Department of Diabetes and Endocrinology, Link 7-C, Royal Liverpool University Hospital, Prescot Street, Liverpool, L7 8XP, UK, \\ ${ }^{2}$ Faculty of Mathematical Sciences, University of Lahore, Raiwind Road, Lahore, Pakistan and ${ }^{3}$ Wansbeck General Hospital, Woodhorn Lane, \\ Ashington, Northumberland, NE63 9JJ, UK
}

(Correspondence should be addressed to A M Ahmad; Email: DRAAHMAD@yahoo.com)

(This work was conducted while Dr A M Ahmad was working at Wansbeck General Hospital, Woodhorn Lane, Ashington, Northumberland, NE63 9JJ, UK)

\begin{abstract}
Objective: Several risk factors have been shown individually to influence the outcome following radioactive iodine (RAI) therapy in the treatment of hyperthyroidism. However, no attempt has been made to determine their independent prognostic values that could be used in a regression model to provide objective estimates of the probability of developing hypothyroidism.

Study design and methods: We audited records of all hyperthyroid patients treated with first dose RAI between 1980 and 1996. Patients were aetiologically categorized into Graves' disease, solitary toxic nodule and toxic multinodular goiter. Following RAI, outcome was categorized as hypothyroidism, euthyroidism and persistent hyperthyroidism. Multiple logistic regression analysis was used to identify significant risk factors, their prognostic values and probability estimates of developing hypothyroidism in the presence of one or more of these factors.

Results: The cumulative incidence of hypothyroidism was $55.8 \%$ at 1 year and $86.1 \%$ at 10 years. Graves' disease (odds ratio: 4.29), presence of thyroid autoantibodies (odds ratio: 3.51), no antithyroid treatment given prior to RAI (odds ratio: 2.50), non-palpable goiter (odds ratio: 2.48) and high RAI dose (odds ratio: 1.90), were identified as significant independent risk factors. We then developed a predictive table that provides objective estimates of developing hypothyroidism. In the absence of all risk factors we can predict an $11.9 \%$ probability of developing hypothyroidism; this increases linearly to a $96.4 \%$ probability in the presence of all factors.

Conclusions: These objective estimates would help in understanding the relative contributions of the known risk factors, and to predict the probability of developing hypothyroidism following RAI treatment. This would not only help patients make an informed consent for a treatment that would lead to life-long replacement therapy but may also prove useful in calculating the RAI dose that may reduce or delay the onset of developing hypothyroidism.
\end{abstract}

European Journal of Endocrinology 146 767-775

\section{Introduction}

Hypothyroidism is a recognised major undesirable side effect of radioactive iodine (RAI) treatment for hyperthyroidism (1-4). Many dosage schedules of RAI, ranging from arbitrary deliberate ablation $(5,6)$ to elaborately calculated doses based on the size of the thyroid gland, uptake of radioiodine or the turnover of radioiodine $(2,6,7)$, have been used with little consensus about the most appropriate dosage regimen. Whilst it is possible to deliver a relatively accurate radiation dose to the thyroid gland, the biological response of the gland remains unpredictable. Although the incidence of early-onset hypothyroidism was reduced with calculated RAI doses, the incidence of late-onset hypothyroidism was disappointingly high $(2,6,7)$. In contrast, large ablative RAI doses result in a high incidence of early-onset hypothyroidism $(5,6)$ and have been recommended by some clinicians to obviate the need for long-term follow-up following such treatment. In view of the possible long-term effects of RAI treatment and thyroxine replacement (8), we need to be careful in administering large RAI doses.

There is sufficient evidence that risk factors other than the RAI dose, such as presence of thyroid antibodies (9, 10), aetiology of hyperthyroidism (11), administration of antithyroid drugs $(12,13)$, and goiter size $(14,15)$, individually influence the outcome 
following RAI treatment. However, studies using calculated RAI doses have failed to take into account the relative contributions of these risk factors whilst calculating the dosage and this may explain the disappointing results. No attempt has been made to determine the independent prognostic values of these risk factors that can be used to provide objective estimates of the probability of developing hypothyroidism. Such estimates may help identify patients who have a significantly higher probability of developing hypothyroidism in the presence of more than one of these risk factors and may only require a small dose of RAI to achieve euthyroidism or hypothyroidism compared with those with a lower probability, thus requiring relatively higher RAI dosage. Accurate, objective estimates would also help clinicians understand the relative contributions of the known risk factors and allow them to predict the probability of developing hypothyroidism following RAI treatment, thus allowing patients to make an informed consent for a treatment that may lead to life-long replacement therapy.

Therefore, in this study we have examined data on the outcome in patients treated with first dose RAI between 1980 and 1996. We then identified the significant risk factors influencing the outcome and their predictive values in a multiple logistic regression model. The aim was to determine objective probability estimates of developing hypothyroidism following RAI and to develop a simple predictability table, thereby reducing reliance on subjective estimates of the outcome.

\section{Materials and methods}

We retrospectively reviewed the records of all 327 hyperthyroid patients who were treated with RAI between 1980 and 1996. The diagnosis of hyperthyroidism was based on clinical features, suppressed thyrotropin (TSH) levels and increased total thyroxine $\left(\mathrm{T}_{4}\right)$, free $\mathrm{T}_{4}$ or free tri-iodothyronine $\left(\mathrm{T}_{3}\right)$. All patients between 1980 and 1988 were treated with a fixed RAI dose of 550 megabequerels (Mbq), as a policy of the nuclear medicine department, that changed to a lower dose of $400 \mathrm{Mbq}$ between 1989 and 1996. Information collected included age, gender, aetiology of hyperthyroidism (Graves' disease, solitary toxic nodule (STN) and multinodular goiter (MNG) based on the nodularity of the goiter, presence or absence of thyroid autoantibodies, ultrasonography of thyroid gland or thyroid iodine uptake scan), thyroid autoantibody status (present or absent; titres of $<1: 100$ were considered absent), antithyroid drug (ATD) treatment prior to RAI (not given or given), goiter (not palpable or palpable; palpation was performed by the same consultant endocrinologist and confirmed by ultrasonography or thyroid iodine uptake scan) and RAI dose (550 Mbq or $400 \mathrm{Mbq}$ ).
Of the 327 patients treated with RAI, 53 patients were excluded from the study; 26 of the 53 patients had a follow-up less than 6 months, 15 had undergone subtotal thyroidectomy prior to RAI, 10 patients were previously treated with RAI elsewhere and in 2 patients the case notes were unavailable. The remaining 274 patients were included in the study.

Following RAI, the outcome was classified as persistent thyrotoxicosis, euthyroidism or hypothyroidism, based on the functional status of the thyroid. Thyroid status was assessed at monthly intervals after RAI treatment. Persistent thyrotoxicosis was diagnosed if free $\mathrm{T}_{4}$ or $\mathrm{T}_{3}$ remained elevated. Patients who remained hyperthyroid at 6 months were retreated with RAI and were considered as single-dose RAI treatment failures. Euthyroidism was diagnosed if patients remained euthyroid while off all treatment for 6 months, including $\mathrm{T}_{4}$ replacement (11). Hypothyroidism was diagnosed if TSH was elevated and serum $\mathrm{T}_{4}$ or $\mathrm{T}_{3}$ was below the normal reference range (16). Thyroid replacement was commenced following confirmation of hypothyroidism. Patients with elevated TSH but normal $\mathrm{T}_{4}$ or with a modest reduction in $\mathrm{T}_{4}$ were treated with thyroid replacement that was later withdrawn to reassess the thyroid status to exclude transient hypothyroidism. Patients were discharged from the hospital outpatient clinic when they became hypothyroid; they then commenced thyroxine replacement, which was then stable for 6 months. Patients receiving antithyroid drug treatment prior to RAI had, in each case, been advised to stop medication 7 days before receiving RAI and the treatment was not recommenced for at least 2 weeks after RAI treatment.

\section{Statistical analysis}

Descriptive statistics are expressed as either means \pm standard deviation (S.D.) or frequencies, as appropriate. Chi-square test was used to determine the significance of the effect of individual variables on the outcome classified as persistent thyrotoxicosis, euthyroidism or hypothyroidism. Forward stepwise logistic regression analysis was performed to evaluate the risk factors and their prognostic significance in the development of hypothyroidism. For regression analysis the dependent (outcome) variable was classified as hypothyroidism or non-hypothyroidism (persistent hyperthyroidism or euthyroidism). Age ( $\leq$ and $>55$ years), gender, Graves' disease or non-Graves' disease (STN and MNG), thyroid autoantibodies (present or absent), ATD treatment prior to RAI (not given or given), goiter (not palpable or palpable) and RAI dose ( $550 \mathrm{Mbq}$ or $400 \mathrm{Mbq}$ ) were used as covariates. The regression coefficient $(\beta)$ was obtained for each individual variable and then used to estimate the odds ratio (OR), using the equation $\mathrm{OR}=e^{\beta}$. The standard error of $\beta$ was used to calculate the $95 \%$ confidence 
interval (95\% CI) of $\beta$ and OR. OR represents the factor by which the risk of hypothyroidism is multiplied for the patients in the presence of the variable.

The cumulative incidence of hypothyroidism was determined by life-table analysis using SPSS statistical package (version 9.0) and the difference in incidence of hypothyroidism between subgroups was compared using Wilcoxon (Gehan) test. A $P$ value of $<0.05$ was considered significant.

\section{Results}

Of the 274 patients (males $=41$, females $=233$; mean age \pm S.D. at the time of RAI was $56.1 \pm 13.0$ years), the diagnosis was Graves' disease in 186 patients, STN in 28 patients and MNG in 60 patients. One hundred and forty-five patients received an RAI dose of $400 \mathrm{Mbq}$ and 129 patients were given $550 \mathrm{Mbq}$. Eighty-one patients were treated with antithyroid drugs (ATD) prior to RAI. Carbimazole was prescribed to 75 patients and propylthiouracil to the other 6 due to intolerability to carbimazole. One hundred and twenty-three patients had positive thyroid autoantibodies and goiter was palpable in 185 patients. One hundred and seventy-five patients developed hypothyroidism following the first dose of RAI and 68 patients remained euthyroid. Thirty-one patients had persistent thyrotoxicosis and required subsequent doses of RAI, following which 16 patients developed hypothyroidism, 8 became euthyroid and 7 patients required a third dose of RAI. Two hundred and one patients were discharged, 51 were still being followedup, 5 had died due to cardiac events and 17 had been lost to follow-up after a mean duration of 54.7 months (range: 18-88 months).

The results of chi-square analysis are presented in Table 1. Hypothyroidism developed in $80.4 \%$ men, 9.8\% became euthyroid and $9.8 \%$ remained hyperthyroid following the first dose of RAI compared with $60.9 \%, 27.5 \%$ and $11.6 \%$ in women respectively $\left(\chi^{2}=6.59, P<0.05\right)$. A higher proportion of patients with Graves' disease developed hypothyroidism (77.4\%) compared with STN (39.3\%) and MNG (33.3\%), whereas the frequency of euthyroidism was significantly higher in STN (50\%) and MNG (50\%) compared with patients with Graves' disease (12.9\%) $\left(\chi^{2}=51.85, P<0.001\right)$. A higher percentage of patients who received an RAI dose of $550 \mathrm{Mbq}$ developed hypothyroidism $(72.1 \%)$, and a lower percentage became euthyroid (19.4\%) or remained hyperthyroid (8.5\%) compared with those who received $400 \mathrm{Mbq}$ $\left(56.6 \%, 29.7 \%\right.$ and $13.7 \%$ respectively; $\chi^{2}=7.15$, $P<0.05)$. The single-dose RAI treatment failure rate was significantly higher $(23.5 \%$ vs $6.2 \%)$ and the frequency of hypothyroidism was lower $(54.3 \%$ vs $67.9 \%$ ) in patients treated with ATD prior to RAI compared with patients who did not receive ATD $\left(\chi^{2}=16.94, P<0.001\right)$. Positive thyroid antibody status and absence of a clinically palpable goiter were associated with a significantly high proportion of patients developing hypothyroidism and a low frequency of single-dose treatment failure rate compared with patients who were antibody negative and had a palpable goiter $\left(\chi^{2}=35.5, P<0.001\right.$ for antibody status; $\chi^{2}=14.34, P<0.001$ for goiter).

The overall cumulative incidence of hypothyroidism and the significance between subgroups according to gender, aetiology of hyperthyroidism, RAI dose, pre-

Table 1 Outcome (number of patients and percentage) following first radioactive iodine dose according to the known risk factors.

\begin{tabular}{|c|c|c|c|c|c|}
\hline & Hypothyroid & Euthyroid & Hyperthyroid & $x^{2 *}$ & $P$ value \\
\hline \multicolumn{6}{|l|}{ Gender } \\
\hline Male $(n=41)$ & $33(80.4 \%)$ & $4(9.8 \%)$ & $4(9.8 \%)$ & & \\
\hline Female $(n=233)$ & $142(60.9 \%)$ & $64(27.5 \%)$ & $27(11.6 \%)$ & 6.59 & $<0.05$ \\
\hline \multicolumn{6}{|l|}{ Aetiology } \\
\hline Graves' $(n=186)$ & $144(77.4 \%)$ & $24(12.9 \%)$ & $18(9.7 \%)$ & & \\
\hline $\operatorname{STN}(n=28)$ & 11 (39.3\%) & $14(50.0 \%)$ & $3(10.7 \%)$ & & \\
\hline $\mathrm{MNG}(n=60)$ & $20(33.3 \%)$ & $30(50.0 \%)$ & $10(16.7 \%)$ & 51.85 & $<0.001$ \\
\hline \multicolumn{6}{|l|}{ Dose of RAI } \\
\hline $550 \mathrm{Mbq}(n=129)$ & $93(72.1 \%)$ & 25 (19.4\%) & $11(8.5 \%)$ & & \\
\hline $400 \mathrm{Mbq}(n=145)$ & $82(56.6 \%)$ & $43(29.7 \%)$ & $20(13.7 \%)$ & 7.15 & $<0.05$ \\
\hline \multicolumn{6}{|l|}{ Pre-treatment with ATD } \\
\hline No ATD $(n=193)$ & $131(67.9 \%)$ & $50(25.9 \%)$ & $12(6.2 \%)$ & & \\
\hline ATD given $(n=81)$ & $44(54.3 \%)$ & $18(22.2 \%)$ & $19(23.5 \%)$ & 16.94 & $<0.001$ \\
\hline \multicolumn{6}{|l|}{ Thyroid antibody } \\
\hline Negative $(n=151)$ & $73(48.3 \%)$ & $55(36.5 \%)$ & $23(15.2 \%)$ & & \\
\hline Positive $(n=123)$ & $102(82.9 \%)$ & $13(10.6 \%)$ & $8(6.5 \%)$ & 35.5 & $<0.001$ \\
\hline \multicolumn{6}{|l|}{ Goiter } \\
\hline Not palpable $(n=89)$ & 70 (78.6\%) & $16(18.0 \%)$ & $3(3.4 \%)$ & & \\
\hline Palpable $(n=185)$ & $105(56.8 \%)$ & $52(28.1 \%)$ & $28(15.1 \%)$ & 14.34 & $<0.001$ \\
\hline
\end{tabular}

${ }^{*}$ Chi-square values for $3 \times 2$ and $3 \times 3$ frequency tables. 
Table 2 Overall cumulative incidence of hypothyroidism and subgroup analysis according to known risk factors*.

\begin{tabular}{|c|c|c|c|c|c|c|}
\hline & 6 months & 1 year & 3 years & 5 years & 10 years & $P$ value \\
\hline Overall & $38.2 \%$ & $55.8 \%$ & $66 \%$ & $69.1 \%$ & $86.1 \%$ & \\
\hline \multicolumn{7}{|l|}{ Gender } \\
\hline Male & $62.5 \%$ & $70.9 \%$ & $80.6 \%$ & $84.5 \%$ & $94.9 \%$ & \\
\hline Female & $32.8 \%$ & $52 \%$ & $63.3 \%$ & $65.9 \%$ & $83.6 \%$ & $<0.001$ \\
\hline \multicolumn{7}{|l|}{ Aetiology } \\
\hline Graves' & $47.1 \%$ & $67.1 \%$ & $78.3 \%$ & $82.3 \%$ & $88.2 \%$ & $<0.001^{\mathrm{a}}$ \\
\hline STN & $4.6 \%$ & $33.6 \%$ & $38.3 \%$ & $38.3 \%$ & $38.3 \%$ & $<0.001^{b}$ \\
\hline MNG & $16 \%$ & $23.8 \%$ & $36.4 \%$ & $36.4 \%$ & $67 \%$ & $n s^{c}$ \\
\hline \multicolumn{7}{|l|}{ Dose of RAI } \\
\hline $550 \mathrm{Mbq}$ & $42.4 \%$ & $57 \%$ & $69.8 \%$ & $73.9 \%$ & $87.6 \%$ & \\
\hline $400 \mathrm{Mbq}$ & $32.6 \%$ & $52.9 \%$ & $62.2 \%$ & $62.2 \%$ & $71.6 \%$ & $<0.01$ \\
\hline \multicolumn{7}{|c|}{ Pre-treatment with ATD } \\
\hline No ATD & $44 \%$ & $60.2 \%$ & $68 \%$ & $71.6 \%$ & $86.4 \%$ & \\
\hline ATD given & $21.3 \%$ & $42.1 \%$ & $62.4 \%$ & $62.4 \%$ & $77.4 \%$ & $<0.01$ \\
\hline \multicolumn{7}{|l|}{ Thyroid antibody } \\
\hline Negative & $25.2 \%$ & $41.3 \%$ & $50.1 \%$ & $54.1 \%$ & $68.7 \%$ & \\
\hline Positive & $51.7 \%$ & $70.9 \%$ & $84.7 \%$ & $86.6 \%$ & $100 \%$ & $<0.001$ \\
\hline \multicolumn{7}{|l|}{ Goiter } \\
\hline Not palpable & $52.9 \%$ & $71.8 \%$ & $83.2 \%$ & $87.4 \%$ & $87.4 \%$ & \\
\hline Palpable & $29.7 \%$ & $46.9 \%$ & $57.7 \%$ & $59.5 \%$ & $81.9 \%$ & $<0.001$ \\
\hline
\end{tabular}

* Comparisons performed using Wilcoxon (Gehan) analysis.

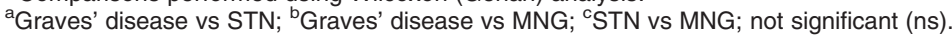

treatment with ATD, thyroid autoantibody status and palpable goiter are detailed in Table 2 and demonstrated in Figs 1 and 2(A-F). The overall cumulative incidence of hypothyroidism following RAI treatment was $38.2 \%$ after 6 months; this increased to $55.8 \%$ after 1 year and was $66 \%, 69.1 \%$, and $86.1 \%$ at the end of 3, 5, and 10 years respectively.

Multiple logistic regression analysis demonstrated that age and gender were not significantly associated with the development of hypothyroidism, whereas Graves' disease $(\mathrm{OR}=4.29)$, positive thyroid autoantibodies $(\mathrm{OR}=3.51)$, no ATD given prior to RAI $(\mathrm{OR}=$ $2.50)$, absence of a palpable goiter $(\mathrm{OR}=2.48)$ and

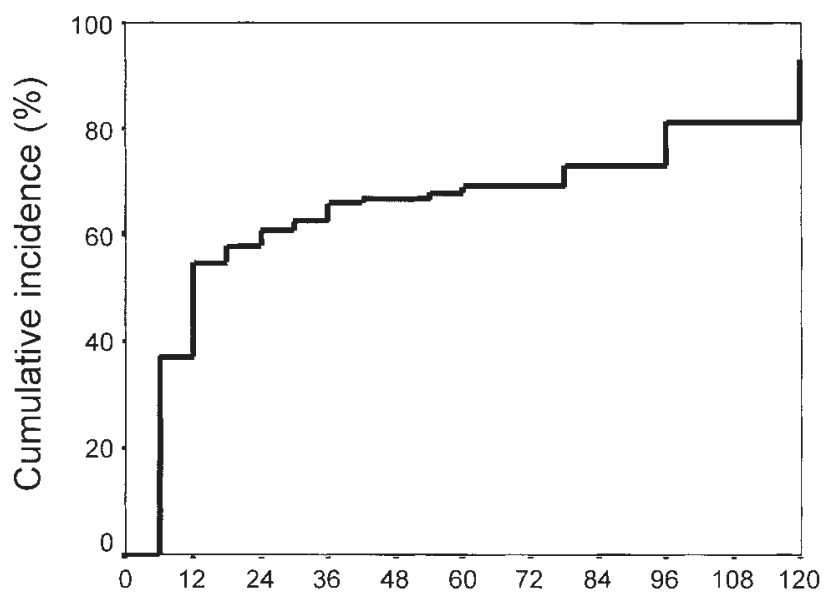

Time to hypothyroidism (months)

Figure 1 Cumulative incidence of hypothyroidism in 274 patients after the first dose of radioactive iodine treatment. higher RAI dose $(\mathrm{OR}=1.90)$ were identified as significant prognostic risk factors following RAI (Table 3). Using the regression coefficients $(\beta)$, we can obtain the predictive value or prognostic index $(y)$ of developing hypothyroidism for any individual following RAI in the presence of one or more identified risk factors by the equation 1 (17):

$$
\text { Logit }-(y)=\beta_{0}+\beta_{1} X_{1}+\beta_{2} X_{2}+\ldots+\beta_{k} X_{k},
$$

where $\beta_{0}$ is the constant $(-2.00)$ and $\beta_{1} \ldots \beta_{k}$ are the regression coefficients of the risk factors $\left(X_{1} \ldots X_{k}\right)$. We would obtain a regression model like the following:

$$
\begin{aligned}
\operatorname{Logit}(y)= & -2.00+1.46 \text { (Graves' disease) } \\
& +1.26 \text { (positive thyroid autoantibodies) } \\
& +0.92 \text { (no pre-treatment with ATD) } \\
& +0.91 \text { (non-palpable goiter) } \\
& +0.64 \text { (high RAI dose) }
\end{aligned}
$$

$X$ or the risk factor would be substituted with numerical value of 1 or 0 based on the presence or absence of the risk factor respectively. The percentage predicted risk $(P)$ or the probability of developing hypothyroidism can then be estimated using the equation 2 (17):

$$
P=\left(e^{y} /\left(1+e^{y}\right)\right) \times 100 .
$$

For example, a patient with Graves' disease and positive thyroid autoantibodies would have a prognostic value of: 
(A)

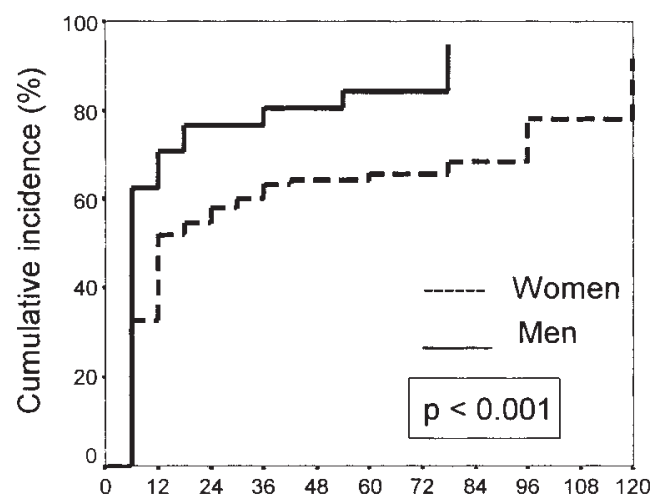

Time to hypothyroidism (months)

(C)

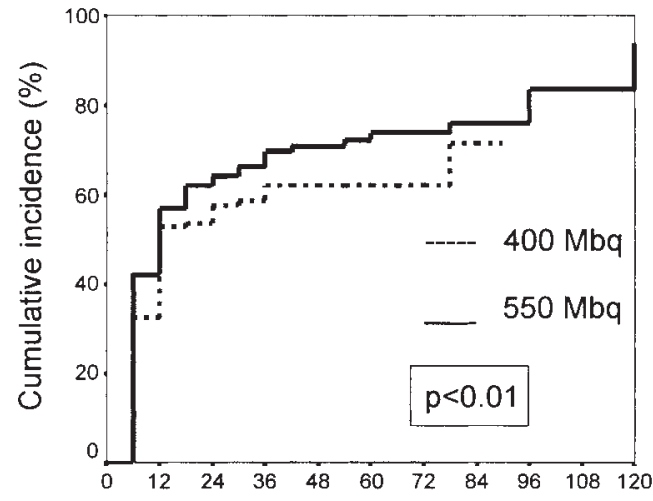

Time to hypothyroidism (months)

(E)

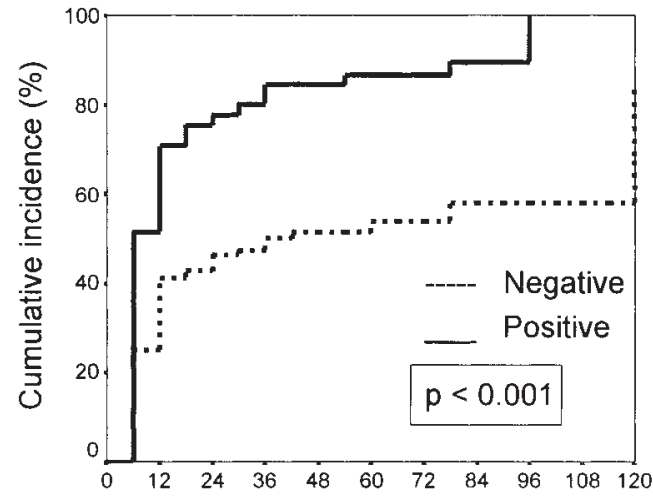

Time to hypothyroidism (months)
(B)

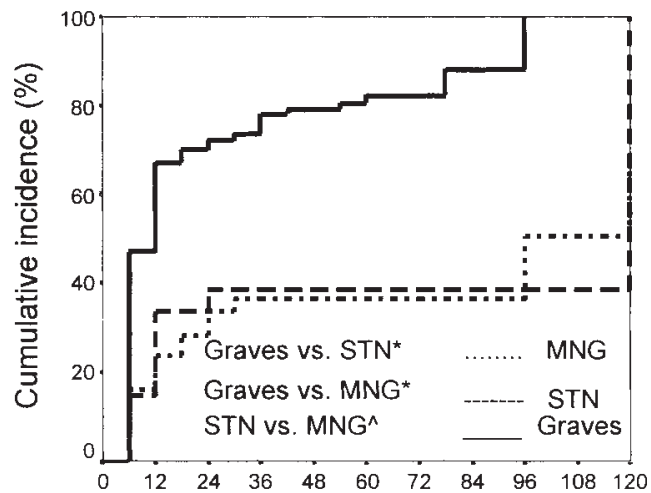

Time to hypothyroidism (months)

(D)

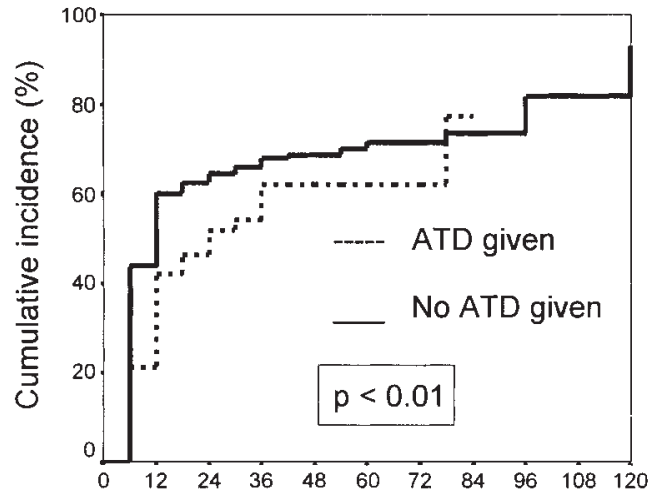

Time to hypothyroidism (months)

(F)

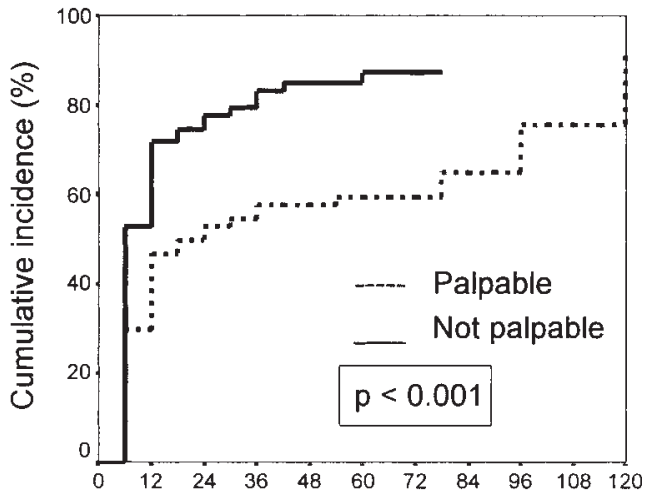

Time to hypothyroidism (months)

Figure 2 Incidence of hypothyroidism according to (A) gender, (B) cause of hyperthyroidism, (C) radioactive iodine dose, (D) antithyroid drug (ATD) treatment prior to radioactive iodine, (E) thyroid autoantibody status, and (F) goiter. ${ }^{\star} P<0.001 ;{ }^{\wedge} P=$ not significant. 
Table 3 Risk factors influencing the outcome following radioactive iodine treatment identified using multiple logistic regression and their predictive value.

\begin{tabular}{lccc}
\hline Factors & $\begin{array}{c}\text { Regression } \\
\text { coefficient }(\beta)\end{array}$ & Odds ratio $(95 \% \mathrm{Cl})^{\mathrm{a}}$ & $\boldsymbol{P}$ value \\
\hline $\begin{array}{l}\text { Aetiology } \\
\text { (Graves' vs non-Graves') }\end{array}$ & 1.46 & $4.29(2.20$ to 8.35$)$ & $<0.001$ \\
$\begin{array}{l}\text { Thyroid antibody status } \\
\text { (positive vs negative) }\end{array}$ & 1.26 & $3.51(1.83$ to 6.73$)$ & $<0.001$ \\
$\begin{array}{l}\text { Pre-treatment with ATD } \\
\text { (not given vs given) }\end{array}$ & 0.92 & $2.50(1.31$ to 4.75$)$ & $<0.01$ \\
$\begin{array}{l}\text { Goiter } \\
\text { (not palpable vs palpable) }\end{array}$ & 0.91 & $2.48(1.23$ to 4.99$)$ & $<0.01$ \\
$\begin{array}{l}\text { Dose of RAl } \\
\text { (550 vs 400 Mbq) }\end{array}$ & 0.64 & $1.90(1.05$ to 3.43$)$ & $<0.05$ \\
Constant $\left(\beta_{0}\right)$ & -2.00 & & \\
\hline
\end{tabular}

a $95 \%$ confidence interval.

$$
\begin{aligned}
\operatorname{Logit}(y)= & -2.00+1.46 \times 1+1.26 \times 1+0.92 \\
& \times 0+0.91 \times 0+0.64 \times 0=0.72,
\end{aligned}
$$

and a percentage predictive risk of:

$$
P=\left(e^{0.72} /\left(1+e^{0.72}\right)\right) \times 100=67.2 \%
$$

to develop hypothyroidism. Similarly, the probability of developing hypothyroidism in the presence of all identified risk factors would be $96.4 \%$. Tables 4 and 5 detail the probability of developing hypothyroidism in the absence or presence of these risk factors in patients with Graves' and non-Graves' disease respectively.

\begin{tabular}{|c|c|c|c|c|c|}
\hline $\begin{array}{l}\text { No. of } \\
\text { risk factors }\end{array}$ & TAb & ATD & Goiter & $\begin{array}{l}\text { RAl } \\
\text { dose }\end{array}$ & $\begin{array}{c}\text { Probability } \\
(\%)\end{array}$ \\
\hline 0 & - ve & $\mathrm{G}$ & P & L & 36.8 \\
\hline 1 & $+\mathrm{ve}$ & G & $\mathrm{P}$ & L & 67.2 \\
\hline 1 & - ve & NG & $\mathrm{P}$ & L & 59.3 \\
\hline 1 & - ve & $\mathrm{G}$ & NP & $L$ & 59.1 \\
\hline 1 & - ve & G & $\mathrm{P}$ & $\mathrm{H}$ & 52.4 \\
\hline 2 & $+\mathrm{ve}$ & $N G$ & $P$ & $L$ & 83.7 \\
\hline 2 & + ve & G & NP & $L$ & 83.6 \\
\hline 2 & $+\mathrm{ve}$ & $\mathrm{G}$ & $\mathrm{P}$ & $\mathrm{H}$ & 79.5 \\
\hline 2 & - ve & NG & NP & $\mathrm{L}$ & 78.4 \\
\hline 2 & - ve & NG & P & $\mathrm{H}$ & 73.4 \\
\hline 2 & - ve & G & NP & $\mathrm{H}$ & 73.3 \\
\hline 3 & $+\mathrm{ve}$ & $\mathrm{NG}$ & NP & $\mathrm{L}$ & 92.7 \\
\hline 3 & + ve & NG & $\mathrm{P}$ & $\mathrm{H}$ & 90.7 \\
\hline 3 & + ve & G & NP & $\mathrm{H}$ & 90.6 \\
\hline 3 & - ve & $N G$ & NP & $\mathrm{H}$ & 87.3 \\
\hline 4 & $+\mathrm{ve}$ & NG & NP & $\mathrm{H}$ & 96.4 \\
\hline
\end{tabular}

Table 4 Probability of developing hypothyroidism in patients with Graves' disease following RAI.

TAb, thyroid antibody status: + ve, positive; - ve, negative. $\mathrm{G}$, given; NG, not given.

P, palpable; NP, not palpable.

$\mathrm{L}$, low dose $(400 \mathrm{Mbq})$; $\mathrm{H}$, high dose $(550 \mathrm{Mbq})$.

\section{Discussion}

Our present study provides information on the independent prognostic significance of the several known risk factors influencing the outcome following RAI and provides a simple predictability table based on the absence or presence of these risk factors. The cumulative incidence of hypothyroidism was $55.8 \%$ at 1 year which increased at an annual rate of 3\%. Graves' disease, positive thyroid autoantibodies, absence of a palpable goiter, no pre-treatment with antithyroid drugs and high RAI dose were identified as significant risk factors with different prognostic values when analyzed in a multivariate model. The risk of developing hypothyroidism, therefore, depends on the number of risk factors present and their individual predictive value prior to RAI. In

\begin{tabular}{|c|c|c|c|c|c|}
\hline $\begin{array}{l}\text { No. of } \\
\text { risk factors }\end{array}$ & TAb & ATD & Goiter & $\begin{array}{l}\text { RAl } \\
\text { dose }\end{array}$ & $\begin{array}{c}\text { Probability } \\
(\%)\end{array}$ \\
\hline 0 & - ve & G & $P$ & L & 11.9 \\
\hline 1 & + ve & $\mathrm{G}$ & $P$ & L & 32.3 \\
\hline 1 & $-v e$ & NG & $\mathrm{P}$ & L & 25.3 \\
\hline 1 & $-\mathrm{ve}$ & $\mathrm{G}$ & NP & $\bar{L}$ & 25.2 \\
\hline 1 & $-\mathrm{ve}$ & G & $\mathrm{P}$ & $\mathrm{H}$ & 20.4 \\
\hline 2 & $+\mathrm{ve}$ & $N G$ & $P$ & L & 54.4 \\
\hline 2 & + ve & G & NP & L & 54.2 \\
\hline 2 & + ve & G & $\mathrm{P}$ & $\mathrm{H}$ & 47.5 \\
\hline 2 & $-\mathrm{ve}$ & $N G$ & NP & L & 45.7 \\
\hline 2 & - ve & NG & P & $\mathrm{H}$ & 39.1 \\
\hline 2 & $-\mathrm{ve}$ & $\mathrm{G}$ & NP & $\mathrm{H}$ & 38.9 \\
\hline 3 & + ve & NG & NP & L & 74.8 \\
\hline 3 & $+\mathrm{ve}$ & NG & $\mathrm{P}$ & $\mathrm{H}$ & 69.4 \\
\hline 3 & + ve & G & NP & $\mathrm{H}$ & 69.2 \\
\hline 3 & - ve & $N G$ & NP & $\mathrm{H}$ & 61.5 \\
\hline 4 & + ve & $N G$ & NP & $\mathrm{H}$ & 84.9 \\
\hline
\end{tabular}

Table 5 Prediction of hypothyroidism in non-Graves' disease (solitary toxic nodule and multinodular goiter) following RAI.

TAb, thyroid antibody status: + ve, positive; - ve, negative.

$\mathrm{G}$, given; NG, not given.

P, palpable; NP, not palpable.

$\mathrm{L}$, low dose (400 Mbq); $\mathrm{H}$, high dose (550 Mbq). 
the absence of any known risk factor the probability of developing hypothyroidism following RAI is greater than 3 times in patients with Graves' disease (36.8\%) compared with patients with STN or MNG ((11.9\%). The risk increases linearly as the number of risk factors increase in both Graves' and non-Graves' disease patients. However, patients with non-Graves' disease have about $30 \%, 20 \%, 15 \%$ and $12 \%$ lower risk in the presence of $1,2,3$ and 4 risk factors respectively (Tables 4 and 5).

One advantage of the objective estimates for the probability of developing hypothyroidism is that they help in understanding the relative influences of specific prognostic risk factors. Although studies have reported antithyroid drug treatment $(2,12,13)$, goiter size $(15$, $18,19)$ and dose of RAI $(5,7,20)$ as contributors to the development of hypothyroidism, few studies have emphasized the contribution of thyroid antibodies on the outcome following RAI (9, 15, 19). Our study emphasizes the degree to which both Graves' disease and the presence of thyroid antibodies contribute to influencing the outcome following RAI and suggests that these two are the strongest predictors of developing hypothyroidism following RAI. Our results and the regression equation would also help identify those patients who are at increased risk of developing hypothyroidism and may, therefore, require a smaller RAI dose compared with those with a lower number of predictive risk factors who have a reduced probability of developing hypothyroidism and may benefit from a larger RAI dose.

The aetiology of hyperthyroidism is an important factor influencing the outcome after RAI treatment. A higher incidence of hypothyroidism has been reported in patients with Graves' disease compared with MNG and STN, whereas a higher rate of single-dose treatment failure was observed in patients with MNG compared with Graves' disease and STN (4). Toxic MNG is relatively resistant to RAI treatment requiring doses higher than widely appreciated and, unlike Graves' disease, hypothyroidism is relatively uncommon (21). There remains controversy regarding the outcome following RAI in patients with STN, with some studies reporting STN to be more radio-resistant compared with Graves' disease (22), whereas others failed to observe an excess of persistent hyperthyroidism in patients with STN (11). In our series of patients, $77.4 \%$ patients with Graves' disease became hypothyroid compared with $39.3 \%$ and $33.3 \%$ with STN and MNG respectively. The incidence of early- and late-onset hypothyroidism was also higher in patients with Graves' disease compared with STN and MNG. However, we found that the single-dose treatment failure rates were similar in these 3 groups, whereas euthyroidism was significantly higher in patients with STN and MNG compared with Graves' disease. The low incidence of hypothyroidism and increased rate of euthyroidism in STN and MNG, observed in our study and in previous studies, may well be due to the fact that the suppressed normal extra nodular tissue is protected by its inability to concentrate RAI. Previous studies have demonstrated a high incidence of hypothyroidism in patients with non-palpable or small goiters $(15,18,19)$. This may, in part, reflect that patients with large goiters are more likely to have MNG as the underlying diagnosis and are at less risk of developing hypothyroidism. It is also possible that patients with small goiters are likely to have received a higher dose of RAI than required and therefore may have a higher risk of developing hypothyroidism. In either case the size of thyroid goiter is an important factor influencing the outcome following RAI.

Evidence suggests that ATD treatment prior to RAI leads to a reduction of early-onset hypothyroidism (2, $23,24)$ and an increased rate of single-dose RAI treatment failure $(25,26)$. These studies and our results suggest a radioprotective effect of ATD treatment when given prior to RAI compared with RAI given alone. ATD of the thiourylene group block the incorporation of iodide into organic form and reduce the thyroid iodine content (27). A contracted intrathyroidal iodine pool may, therefore, reduce the biological half-life of the RAI. Thus, the effect of ATD may be due to the lower radiation dose delivered to the thyroid gland from the given dose of RAI and may suggest a radioprotective influence of ATD treatment when given prior to RAI. There remains controversy about the effects of thyroid antibodies on the outcome following RAI treatment $(2,9,15,19)$. Since RAI treatment triggers a humoral (28) and possibly a cell-mediated autoimmune response within the thyroid gland, it is quite likely that the coexisting chronic thyroiditis represented by the presence of thyroid autoantibodies (29) contributes to the progressive development of hypothyroidism. Our study supports this concept and demonstrates the prognostic significance of the presence of thyroid antibodies on the development of hypothyroidism following RAI therapy.

The ideal dose of RAI that would render patients euthyroid, avoiding a high incidence of hypothyroidism and single-dose RAI treatment failure rate, remains elusive. It can be argued that ablative RAI therapy may be preferable in treating patients with Graves' disease, as the single-dose RAI treatment failure rate is reported to be higher in patients treated with low or calculated RAI doses, whereas the incidence of hypothyroidism is similar in the long-term $(2,3,30)$. It is, however, important to point out that the reported single-dose RAI treatment failure rate ranges from $6 \%$ to as high as $33 \%$ in patients treated with large ablative RAI doses $(4,5,31)$. Furthermore, caution is required in administering large RAI doses in view of the possible long-term effects of RAI and $\mathrm{T}_{4}$ replacement therapy (8). It is possible that the rough approximation of the formulae used to calculate RAI dosage because of the difficulty in assessing 'active' mass of the thyroid in vivo, and the marked non-uniformity of uptake within 
the gland, in addition to individual variation in iodine kinetics, may explain the discrepancies observed in studies using low dose RAI. However, the wide variation of results previously observed using both high and low dose RAI $(2-5,30,31)$ suggests that factors other than RAI dosage influence the outcome following RAI treatment and should be taken into account when calculating RAI dose. Our study emphasizes the degree to which these risk factors influence the outcome and provides a model that could be useful in identifying patients with either a high or a low risk of developing hypothyroidism. This information could be extremely useful in calculating an appropriate RAI dose to achieve either euthyroidism or hypothyroidism.

In conclusion, we have identified significant risk factors for developing hypothyroidism and using their independent predictive values we have developed a simple table that provides objective rather than subjective estimates to predict the outcome following RAI. Our study may explain the variable outcome previously observed following RAI therapy and may account for the high rate of reported single-dose RAI treatment failure. We suggest that clinicians and nuclear medicine departments use this model and probability estimates to predict the risk of developing hypothyroidism and to calculate the dose of RAI depending on the presence or absence of one or more of these risk factors. Future studies using this paradigm may be able to demonstrate a method to devise an ideal dose of RAI that may render patients euthyroid with a reduced single-dose RAI treatment failure rate and incidence of hypothyroidism. This may reduce the use of life-long $\mathrm{T}_{4}$ replacement therapy and repeated doses of RAI, which may prove highly cost effective.

\section{References}

1 Wise PH, Burnet RB, Ahmad A \& Harding PE. Intentional radioiodine ablation in Graves' disease. Lancet 19752 1231-1233.

2 Sridama V, McCormick M, Kaplan EL, Fauchet R \& DeGroot LJ. Long-term follow-up study of compensated low-dose ${ }^{131}$ I therapy for Graves' disease. New England Journal of Medicine 1984311 $426-432$.

3 Goolden AW \& Stewart JS. Long-term results from graded low dose radioactive iodine therapy for thyrotoxicosis. Clinical Endocrinology 198624 217-222.

4 Bertelsen J, Herskind AM, Sprogøe Jakobsen U \& Hegedüs L. Is standard $555 \mathrm{MBq}{ }^{131}$ I-therapy of hyperthyroidism ablative? Thyroidology 19924 103-106.

5 Kendall-Taylor P, Keir MJ \& Ross WM. Ablative radioiodine therapy for hyperthyroidism: long term follow up study. British Medical Journal 1984289 361-363.

6 Jarløv AE, Hegedüs L, Kristensen LO, Nygaard B \& Hansen JM. Is calculation of the dose in radioiodine therapy of hyperthyroidism worth while? Clinical Endocrinology 199543 325-329.

7 Franklyn JA, Daykin J, Drolc Z, Farmer M \& Sheppard MC. Longterm follow-up of treatment of thyrotoxicosis by three different methods. Clinical Endocrinology 199134 71-76.

8 Franklyn JA, Maisonneuve P, Sheppard M, Betteridge J \& Boyle P. Cancer incidence and mortality after radioiodine treatment for hyperthyroidism: a population-based cohort study. Lancet 1999 $3532111-2115$.

9 Lundell G \& Holm LE. Hypothyroidism following ${ }^{131}$ I therapy for hyperthyroidism in relation to immunologic parameters. Acta Radiologica. Oncology $198019449-454$.

10 DeGroot LJ, Mangklabruks A \& McCormick M. Comparison of RA ${ }^{131}$ I treatment protocols for Graves' disease. Journal of Endocrinological Investigation 199013 111-118.

11 Franklyn JA, Daykin J, Holder R \& Sheppard MC. Radioiodine therapy compared in patients with toxic nodular or Graves' hyperthyroidism. Quarterly Journal of Medicine $1995 \mathbf{8 8}$ $175-180$.

12 Koroscil TM. Thionamides alter the efficacy of radioiodine treatment in patients with Graves' disease. Southern Medical Journal $199588831-836$.

13 Sabri O, Zimny M, Schulz G, Schreckenberger M, Reinartz P, Willmes $\mathrm{K}$ et al. Success rate of radioiodine therapy in Graves' disease: the influence of thyrostatic medication. Journal of Clinical Endocrinology and Metabolism 199984 1229-1233.

14 Nofal MM, Beierwaltes WH \& Patno ME. Treatment of hyperthyroidism with sodium iodide I-131. A 16-year experience. Journal of the American Medical Association 1966197 605610.

15 Marcocci C, Gianchecchi D, Masini I, Golia F, Ceccarelli C, Bracci E et al. A reappraisal of the role of methimazole and other factors on the efficacy and outcome of radioiodine therapy of Graves' hyperthyroidism. Journal of Endocrinological Investigation 1990 13 513-520.

16 Allahabadia A, Daykin J, Holder RL, Sheppard MC, Gough SC \& Franklyn JA. Age and gender predict the outcome of treatment for Graves' hyperthyroidism. Journal of Clinical Endocrinology and Metabolism 200085 1038-1042.

17 Altman DG. Relation between several variables. In Practical Statistics for Medical Research, pp 351-358. Ed. DG Altman. London: Chapman \& Hall, 1995.

18 Blomfield GW, Eckert H, Fisher M, Miller MA, Munro DS \& Wilson GM. Treatment of thyrotoxicosis with ${ }^{131}$ I. British Medical Journal 1959163.

19 Latapie JL, Lefort G, Commenges M, Roger P, Riviere LJ \& Mauriac L. The utilization of small repeated doses of iodine 131 in the treatment of Graves' disease. Results. Annales d'Endocrinologie $198041601-605$.

20 Allahabadia A, Daykin J, Sheppard MC, Gough SC \& Franklyn JA. Radioiodine treatment of hyperthyroidism - prognostic factors for outcome. Journal of Clinical Endocrinology and Metabolism 200186 3611-3617.

21 Hamburger JI \& Hamburger SW. Diagnosis and management of large toxic multinodular goiters. Journal of Nuclear Medicine $198526888-892$.

22 Farrar JJ \& Toft AD. Iodine-131 treatment of hyperthyroidism: current issues. Clinical Endocrinology 199135 207-212.

23 Reynolds LR \& Kotchen TA. Antithyroid drugs and radioactive iodine. Fifteen years' experience with Graves' disease. Archives of Internal Medicine 1979139 651-653.

24 Steinbach JJ, Donoghue GD \& Goldman JK. Simultaneous treatment of toxic diffuse goiter with I-131 and antithyroid drugs: a prospective study. Journal of Nuclear Medicine 197920 1263-1267.

25 Tuttle RM, Patience T \& Budd S. Treatment with propylthiouracil before radioactive iodine therapy is associated with a higher treatment failure rate than therapy with radioactive iodine alone in Graves' disease. Thyroid 19955 243-247.

26 Hancock LD, Tuttle RM, LeMar H, Bauman J \& Patience T. The effect of propylthiouracil on subsequent radioactive iodine therapy in Graves' disease. Clinical Endocrinology $1997 \mathbf{4 7}$ 425-430.

27 Barandes M, Hurley JR \& Becker DV. Implications of rapid intrathyroidal iodine turnover for ${ }^{131} \mathrm{I}$ therapy: The small pool syndrome. Journal of Nuclear Medicine 197314379.

28 Pinchera A, Liberti P, Martino E, Fenzi GF, Grasso L, Baschieri L et al. Effect of antithyroid therapy on the long-acting thyroid 
stimulator and antithyroglobulin antibodies. Journal of Clinical Endocrinology and Metabolism 196929 231-8.

29 Doniach D. Humoral and genetic aspects of thyroid autoimmunity. Clinical Endocrinology and Metabolism 19754267.

30 Watson AB, Brownlie BE, Frampton CM, Turner JG \& Rogers TG. Outcome following standardized $185 \mathrm{MBq}$ dose ${ }^{131} \mathrm{I}$ therapy for Graves' disease. Clinical Endocrinology 198828 487-496.
31 Ratcliffe GE, Fogelman I \& Maisey MN. The evaluation of radioiodine therapy for thyroid patients using a fixed-dose regime. British Journal of Radiology 198659 1105-1107.

Received 6 November 2001

Accepted 21 March 2002 